\title{
ARTICLE OPEN Detection of an anti-angina therapeutic module in the effective population treated by a multi-target drug Danhong injection: a randomized trial
}

Jun Liu' ${ }^{1}$, Dan-Dan Liं ${ }^{2}$, Wei Dong ${ }^{2}$, Yu-Qi Liư ${ }^{2}$, Yang Wu ${ }^{3}$, Da-Xuan Tang ${ }^{3}$, Fu-Chun Zhang ${ }^{4}$, Meng Qiu ${ }^{4}$, Qi Hua ${ }^{5}$, Jing-Yu He ${ }^{5}$, Jun Li ${ }^{6}$, Bai Du ${ }^{6}$, Ting-Hai Du${ }^{7}$, Lin-Lin Niu ${ }^{7}$, Xue-Jun Jiang ${ }^{8}$, Bo Cui ${ }^{8}$, Jiang-Bin Chen ${ }^{8}$, Yang-Gan Wang ${ }^{9}$, Hai-Rong Wang ${ }^{9}$, Qin $\mathrm{Yu}^{10}{ }^{10}$ Jing $\mathrm{He}^{10}$, Yi-Lin Mao ${ }^{11}$, Xiao-Fang Bin ${ }^{11}$, Yue Deng ${ }^{12}$, Yu-Dan Tian ${ }^{12}$, Qing-Hua Han ${ }^{13}$, Da-Jin Liu ${ }^{13}$, Li-Qin Duan ${ }^{13}$, Ming-Jun Zhao ${ }^{14}$, Cui-Ying Zhang ${ }^{14}$, Hai-Ying Dai ${ }^{15}$, Ze-Hua $\mathrm{Li}^{15}$, Ying Xiao ${ }^{15}$, You-Zhi Hu ${ }^{16}$, Xiao-Yu Huang ${ }^{16}$, Kun Xing ${ }^{17}$, Xin Jiang ${ }^{17}$, Chao-Feng Liu ${ }^{18}$, Jing $\mathrm{An}^{18}$, Feng-Chun $\mathrm{Li}^{19}{ }^{19}$, Tao Tao ${ }^{19}$, Jin-Fa Jiang ${ }^{20}$, Ying Yang ${ }^{20}$, Yao-Rong Dong ${ }^{21}$, Lei Zhang ${ }^{21}$, Guang $\mathrm{Fu}^{22}$, Ying $\mathrm{Li}^{22}$,

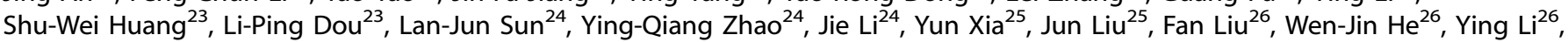
Jian-Cong Tan ${ }^{27}$, Yang Lin ${ }^{27}$, Ya-Bin Zhou ${ }^{28}$, Jian-Fei Yang ${ }^{28}$, Guo-Qing Ma ${ }^{29}$, Hui-Jun Chen ${ }^{29}$, He-Ping Liu ${ }^{30}$, Zong-Wu Liu ${ }^{30}$, Jian-Xiong Liu ${ }^{31}$, Xiao-Jia Luo ${ }^{31}$, Xiao-Hong Bin ${ }^{31}$, Ya-Nan Yu', Hai-Xia Dang ${ }^{1,32}$, Bing Li $\mathrm{Li}^{1,33}$, Fei Teng $\mathbb{B}^{34}$, Wang-Min Qiao ${ }^{34}$, Xiao-Long Zhu ${ }^{34}$, Bing-Wei Chen ${ }^{35}$, Qi-Guang Chen ${ }^{35}$, Chun-Ti Shen ${ }^{36}$, Yong-Yan Wang ${ }^{1 凶}$, Yun-Dai Chen ${ }^{2 凶}$ and Zhong Wang ${ }^{1 凶}$

It's a challenge for detecting the therapeutic targets of a polypharmacological drug from variations in the responsed networks in the differentiated populations with complex diseases, as stable coronary heart disease. Here, in an adaptive, 31-center, randomized, doubleblind trial involving 920 patients with moderate symptomatic stable angina treated by 14-day Danhong injection(DHI), a kind of polypharmacological drug with high quality control, or placebo ( $0.9 \%$ saline), with 76 -day following-up, we firstly confirmed that DHI could increase the proportion of patients with clinically significant changes on angina-frequency assessed by Seattle Angina Questionnaire $(\triangle \mathrm{SAQ}-\mathrm{AF} \geq 20)(12.78 \%$ at Day $30,95 \%$ confidence interval $[\mathrm{Cl}] 5.86-19.71 \%, P=0.0003,13.82 \%$ at Day $60,95 \% \mathrm{Cl} 6.82-20.82 \%, P=0.0001$ and $8.95 \%$ at Day $90,95 \% \mathrm{Cl} 2.06-15.85 \%, P=0.01)$. We also found that there were no significant differences in new-onset major vascular events $(P=0.8502)$ and serious adverse events $(P=0.9105)$ between $\mathrm{DH}$ and placebo. After performing the RNA sequencing in 62 selected patients, we developed a systemic modular approach to identify differentially expressed modules (DEMs) of $D H I$ with the $Z_{\text {summary }}$ value less than 0 compared with the control group, calculated by weighted gene co-expression network analysis (WGCNA), and sketched out the basic framework on a modular map with 25 functional modules targeted by DHI. Furthermore, the effective therapeutic module (ETM), defined as the highest correlation value with the phenotype alteration ( $\triangle \mathrm{SAQ}-\mathrm{AF}$, the change in SAQ-AF at Day 30 from baseline) calculated by WGCNA, was identified in the population with the best effect $(\triangle S A Q-A F \geq 40)$, which is related to anticoagulation and regulation of cholesterol metabolism. We assessed the modular flexibility of this ETM using the global topological D value based on Euclidean distance, which is correlated with phenotype alteration $\left(r^{2}: 0.8204, P=0.019\right)$ by linear regression. Our study identified the anti-angina therapeutic module in the effective population treated by the multi-target drug. Modular methods facilitate the discovery of network pharmacological mechanisms and the advancement of precision medicine. (ClinicalTrials.gov identifier: NCT01681316).

Signal Transduction and Targeted Therapy (2021)6:329

; https://doi.org/10.1038/s41392-021-00741-x

\begin{abstract}
'Institute of Basic Research in Clinical Medicine, China Academy of Chinese Medical Sciences, Beijing, China; ${ }^{2}$ Department of Cardiology, Chinese PLA General Hospital, Beijing, China; ${ }^{3}$ Department of Cardiology, Dongfang Hospital Affiliated to Beijing University of Chinese Medicine, Beijing, China; ${ }^{4}$ Department of Geratology, Peking University Third Hospital, Beijing, China; ${ }^{5}$ Department of Cardiology, Xuan Wu Hospital, Capital Medical University, Beijing, China; ${ }^{6}$ Department of Cardiology, Guang'anmen Hospital, China Academy of Chinese Medical Sciences, Beijing, China; ${ }^{7}$ Department of Cardiology, First Affiliated Hospital of Henan University of Chinese Medicine, Zhengzhou, Henan, China; ${ }^{8}$ Department of Cardiology, Wuhan University Renmin Hospital, Wuhan, Hubei, China; ${ }^{9}$ Department of Cardiology, Wuhan University Zhongnan Hospital, Wuhan, Hubei, China; ${ }^{10}$ Affiliated Zhongshan Hospital of Dalian University, Dalian, Liaoning, China; ${ }^{11}$ Department of Cardiology, Second Affiliated Hospital to Hunan University of Chinese Medicine, Changsha, Hunan, China; ${ }^{12}$ Department of Cardiology, First Affiliated Hospital to Changchun University of Chinese Medicine, Changchun, Jilin, China; ${ }^{13}$ Department of Cardiology, First Affiliated Hospital to Shanxi Medical University, Taiyuan, Shanxi, China; ${ }^{14}$ Department of Cardiology, Affiliated Hospital of Shanxi University of Chinese Medicine, Xianyang, Shanxi, China; ${ }^{15}$ Department of Cardiology, Changsha Central Hospital, Changsha, Hunan, China; ${ }^{16}$ Department of Cardiology, Hubei Provincial Hospital of Traditional Chinese Medicine, Wuhan, Hubei, China; ${ }^{17}$ Department of Cardiology, Shanxi Provincial People's Hospital, Xi'an, Shanxi, China; ${ }^{18}$ Department of Cardiology, Shanxi Province Hospital of Traditional Chinese Medicine, Xi'an, Shanxi, China; ${ }^{19}$ Department of Cardiology, Xi'an City Hospital of Traditional Chinese Medicine, Xi'an, Shanxi, China; ${ }^{20}$ Department of Cardiology, Shanghai Tongji Hospital, Shanghai, China; ${ }^{21}$ Department of Cardiology, Shanghai Municipal Hospital of Traditional Chinese Medicine, Shanghai, China; ${ }^{22}$ Department of Cardiology, The First Hospital of Changsha, Changsha, Hunan, China; ${ }^{23}$ Department of Cardiology, Xinhua Hospital of Zhejiang Province, Hangzhou, Zhejiang, China; ${ }^{24}$ Department of Cardiology, Second Affiliated Hospital of Tianjin University of Traditional Chinese Medicine, Zengchan Dao, Tianjin, China; ${ }^{25}$ Department of Chinese medicine, Shanghai Tenth People's Hospital, Shanghai, China; ${ }^{26}$ Department of Cardiology, Chongqing City Hospital of Traditional Chinese Medicine, Chongqing, China; ${ }^{27}$ Department of Cardiology, Third People's Hospital of Chongqing, Chongqing, China; ${ }^{28}$ Department of Cardiology, First Affiliated Hospital of Heilongjiang University of Traditional Chinese Medicine, Harbin, Heilongjiang, China; ${ }^{29}$ Department of Cardiology, Second Affiliated Hospital of Heilongjiang University of Traditional Chinese Medicine, Harbin, Heilongjiang, China; ${ }^{30}$ Department of Cardiology, Jilin Province People's Hospital, Changchun, Jilin, China; ${ }^{31}$ Department of Cardiology, Chengdu Second People's Hospital, Chengdu, Sichuan, China; ${ }^{32}$ China Academy of Chinese Medical Sciences, Beijing, China; ${ }^{33}$ Institute of Chinese Meteria Medica, China Academy of Chinese Medical Sciences, Beijing, China; ${ }^{34}$ Beijing Genomics Institute (Shenzhen), Shenzhen, Guangdong, China; ${ }^{35}$ School of Public Health, Southeast University, Nanjing, Jiangsu, China and ${ }^{36}$ Changzhou Hospital of Traditional Chinese Medicine, Changzhou, Jiangsu, China
\end{abstract}

Correspondence: Yong-Yan Wang (wangyongyan2010@sina.cn) or Yun-Dai Chen (cyundai@medmail.com.cn) or Zhong Wang (zhonw@vip.sina.com)

These authors contributed equally: Jun Liu, Dan-Dan Li

Received: 29 March 2021 Revised: 11 August 2021 Accepted: 16 August 2021

Published online: 01 September 2021 


\section{INTRODUCTION}

Chronic stable angina is a main manifestation in approximately half of all patients who present with coronary artery disease (CAD), which is a leading cause of death worldwide. ${ }^{1}$ With the realization that CAD involves perturbations of large complex biological networks, ${ }^{2}$ future success in managing $C A D$ may shift from "reactive medicine", which generally treats individual targeted items, e.g., LDL-C, to "proactive P4 medicine" (predictive, preventive, personalized, and participatory), in which disease mechanisms and systems biology allow for a multitargeted and patient-centric approach. ${ }^{3-5}$ However, for complex CAD interventions, particularly through polypharmacological drugs with multitargets, it is especially difficult to detect the specific targets for the matched population. In the network pharmacology, the multitargets on CAD or angina have been identified from the differentially expressed genes ${ }^{6,7}$ to the subnetwork with correlative genes. ${ }^{8-10}$ Therefore, in our study, the certain correlation between CAD and polypharmacological drug treatment should be evaluated with modular pharmacology beyond network pharmacology, which can connect the biological network to the pharmacological network based on the minimal functional unit modules. ${ }^{11}$ Modular flexibility can be adapted to different disease conditions in different people, and the various modular units themselves can be customized in a variety of ways to accommodate different interventions. ${ }^{12}$ Subsequently, it is possible to identify the effective therapeutic module of a multi-target drug in the responded population.

Danhong injection (DHI, made by Shandong Danhong Pharmaceutical Co. Ltd., with quality control via high-performance liquid chromatography (HPLC) fingerprinting (Supplementary Fig. S1) for over $90 \%$ similarity among the batches used in this trial) is a polycomponent drug, which contains 5 main compounds including Danshensu sodium, protocatechualdehyde, p-coumaric acid, rosmarinci acid, salvianolic acid B (Supplementary Fig. S2), exacted from two kind of herbal medicine, Danshen and Honghua. It is widely used in China due to its safety and efficacy in treating cardiovascular diseases. ${ }^{13,14} \mathrm{DHI}$ was reported to have multiple pharmacological effects with multiple targets on $C A D,{ }^{14}$ including promoting endothelial progenitor cells (EPCS) mobilization via upregulating the expression of Akt, eNOS and MMP-9, ${ }^{15}$ attenuating atherosclerosis and macrophage lipid accumulation by promoting the activation of PI3K/AKT insulin signaling pathway, ${ }^{16}$ reducing the platelet aggregation ${ }^{17}$ as well as reducing oxidative stress and maintaining mitochondrial integrity via the Keap1/Nrf2/ JNK pathway. ${ }^{18}$ Meanwhile, the main compounds, as Danshensu sodium, and salvianolic acids were also reported with polypharmacological effects against platelet aggregation and thrombus formation, promoting fibrin degradation, protecting against myocardial ischemia, and improving the microcirculation. ${ }^{19,20}$ Furthermore, a number of clinical studies also demonstrated that $\mathrm{DHI}$ might be an effective and safe treatment option to prevent angina attacks in the management of CAD. ${ }^{13,21}$ However, prior clinical studies generally provided low-quality evidence, and the molecular features of the targeted populations remained to be uncovered. Therefore, in this study, we conducted a welldesigned, randomized, multicenter, double-blind, placebocontrolled trial with a prespecific selected 62-case RNA sequencing study to investigate the effect, safety and effective therapeutic module of $\mathrm{DHI}$ among patients with chronic stable angina.

\section{RESULTS}

Danghong Injection could improve the angina-specific health status with favorable safety

From December 2012 through October 2016, we screened 1327 patients at 31 centers in China; 615 eligible patients were randomly assigned to the $\mathrm{DHI}$ group, while 305 were assigned to the control group. Ultimately, 918 patients diagnosed with CAD using coronary angiography or computed tomography angiography were included in the "intention-to-treat" population for efficacy analysis, comprising 613 in the $\mathrm{DHI}$ group and 305 in the control group, since 2 in the $\mathrm{DHI}$ group received duplicate random numbers (Fig. 1). The distributions of demographic and clinical characteristics were reasonably well balanced between the two groups (Supplementary Table S1). The concomitant medications between the two groups during the 90 days showed no significance $(P>0.05)$ (Supplementary Table S2).

Significant differences were observed in the proportions of patients with clinically significant changes on the domain "angina frequency" in Seattle Angina Questionnaire (SAQ-AF) ${ }^{22}$ at Day 30 (DHI vs. placebo: $52.41 \%$ vs. $39.62 \% ; P=0.0003$; difference and its $95 \%$ Cl: $12.78 \%$ (5.86\%, 19.71\%)), Day 60 (60.85\% vs. $47.04 \% ; P=$ 0.0001 ; difference and its $95 \% \mathrm{Cl}: 13.82 \%(6.82 \%, 20.82 \%))$ and Day $90(66.10 \%$ vs. $57.14 \% ; P=0.0100$; difference and its $95 \% \mathrm{Cl}: 8.95 \%$ $(2.06 \%, 15.85 \%)$ ) (Fig. 2a and Supplementary Table S3). According to the three sensitivity analyses, the results of unadjusted and adjusted for center effects showed center factor did not affect the treatment effects, and the results were all robust to departure from missing at random missing at random or to use the mixedeffect model with repeated measures method (Supplementary Table S4). Compared with the placebo group, an increased proportion of patients in the DHI group had clinically significant improvements in physical limitation (PL), treatment satisfaction (TS), and quality of life $(\mathrm{QL})$ from Day 30 through Day $90(P<0.01)$, but in angina stability (AS), these differences were no longer significant at Day $90(P=0.0927)$ (Fig. 2b-e and Supplementary Table S3). We also found that $18.2 \%$ of patients became free of angina (i.e., had a SAQ-AF score of 100) in the DHI group during the first 30 days, with continuous improvement to $34.59 \%$ thereafter at Day 90, which was significantly higher than the proportion in the control group from Day 30 through Day 90 $(P=0.0219-0.0057)$ (Fig. 2f and Supplementary Table S5). Moreover, the results of the subgroup analysis were generally consistent with those obtained from the entire study population (Supplementary Fig. 2g). In all domains of the SAQ, the scores were consistently higher in the $\mathrm{DHI}$ group than in the control group at all posttreatment time points $(P<0.0001 \sim P=0.0193)$ (Supplementary Table S6, Fig. S3).

More dramatic improvements in the Xueyu-Zheng score ${ }^{23}$ were observed in the DHI group than in the control group from Day 7 ( $P$ $=0.0165)$ to Day $90(P<0.0001)$ (Fig. 3a and Supplementary Table S7). We also found that the proportion of patients with significant improvements in the Xueyu-Zheng score was significantly higher in the $\mathrm{DHI}$ group than in the control group from Day 7 to Day $90 .(P \leq$ 0.0001 , Fig. $3 \mathrm{~b}$ and Supplementary Table S7). According to the patients' diaries, the change in the cumulative incidence density of angina was significantly greater in the $\mathrm{DHI}$ group than in the placebo group, beginning from the second week (Days 8-14) (DHI vs. placebo: 0.18 attacks/person-day vs. 0.20 attacks/person-day, $P=$ $0.0272)$ until Day $90(P<0.0001$, Fig. $3 c$ and Supplementary Table S8), and a similar trend was observed in the cumulative consumption density of nitroglycerin from Day 15 to Day $90(P<0.0001)$ (Fig. $3 \mathrm{~d}$ and Supplementary Table S8). The proportion of patients with improvement in Canadian Cardiovascular Society (CCS) class also increased in both groups, but significant differences between the two groups were observed at Day 60 and Day $90(P=0.0087$ and 0.0214 , respectively, Fig. 3e and Supplementary Table S9). Significant differences in the mean triglyceride level at Day 14 were observed between the two groups in the population with a triglyceride level $\geq 2.6 \mathrm{mmol} / \mathrm{L}(P=0.0489$, Fig. $3 f)$. However, no significant differences were found between the two groups regarding the proportion of patients with normal electrocardiogram (ECG) recordings (Supplementary Table S9), total cholesterol, high-density lipoprotein (HDL) cholesterol, low density lipoprotein (LDL) cholesterol, populationwide triglyceride, hypersensitive C-reactive protein (hs-CRP), 


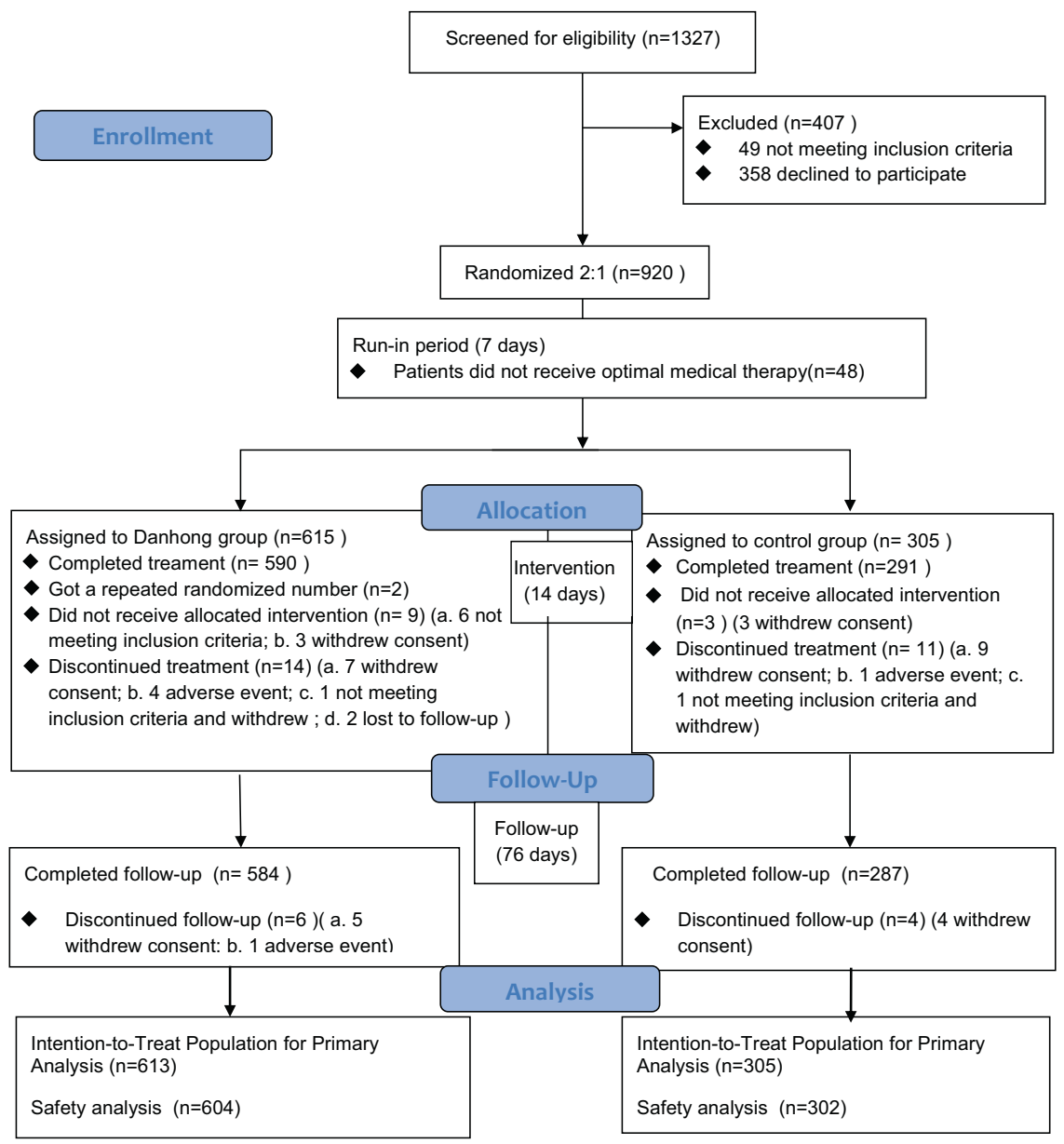

Fig. 1 The flow chart of the trial

C-reactive protein (CRP), or platelet aggregation rate (PAR) at Day 14 $(P=0.1222-0.9495)$ (Supplementary Table S10).

Among the 906 patients who received the study drug, 60 patients reported 93 adverse events (AEs) in the DHI group, and 36 patients reported $58 \mathrm{AEs}$ in the control group; the incidence of $\mathrm{AEs}$ did not differ significantly between the two groups $(P=0.3597)$, and the same was true of the incidence of severe adverse events (SAEs) $(P=0.9105)$. In addition, 4 patients reported new-onset major vascular events within 90 days $(0.5 \%)$ in the $\mathrm{DHI}$ group and $1(0.3 \%)$ in the control group $(P=0.8502)$, and no severe or moderate hemorrhages or deaths were reported within 90 days (Supplementary Table S11).

The changes of differentially expressed genes did not reflect the perturbation of the network by the multi-target drug

Sixty-two patients agreed to provide serum samples for RNA sequencing (28 from 301 Hospital and 34 from Xuanwu Hospital; their clinical characteristics are shown in Supplementary Table S12), including 41 from the DHI group and 21 from the control group. There were 125 differentially expressed genes (DEGs) (75 upregulated and 50 downregulated) in response to DHI treatment, of which 104 mRNAs and 21 non-coded miRNAs (Day 14 or Day 30) (Fig. 4a and Supplementary Table S13), compared with the control group. The enriched GO biological processes (GO-BPs) of these 104 mRNAs were not related to CAD (Supplementary Fig. S4). Eleven DEGs, including 8 mRNAs and 3 miRNAs, were significantly expressed on both Day 14 and Day 30 (Fig. 4b). However, none of these common DEGs was reported to be associated with stable angina. Furthermore, among the 21
miRNAs, 15 were mapped onto identified names via miRBase, ${ }^{24}$ and the 6 novel miRNAs were predicted by miRDeep $2 .{ }^{25}$ We identified 14807 genes as the predicted target genes regulated by these 15 miRNAs via TargetScan Release $7.2,{ }^{26}$ and 44 overlapped with the 104 mRNAs encoded by the DEGs (Fig. 4c); these 44 genes were identified by Metascape ${ }^{27}$ as related to forebrain development (GO: 0030900) and neuron projection extension (GO: 1990138) (Fig. 4d), meaning that they were not likely to be functionally related to stable angina. Here, we found that the changes of DEGs did not reflect the perturbation of the network targeted by $\mathrm{DHI}$.

The multi-target drug Danhong injection treated the angina through a 25-targeted-functional module (TFM) network Using WGCNA, ${ }^{28}$ we detected 38 modules from the coexpressed gene network in the DHI group at Day 30 (Fig. 5a), and then identified 25 differentially expressed modules (DEMs) with $Z_{\text {summary }}$ values ${ }^{29}$ less than 0 for $\mathrm{DHI}$ compared to placebo (Fig. $5 \mathrm{~b}$ and Supplementary Table S14), as the targeted modules of DHI. Then, the targeted module network of DHI was constructed, with 25 nodes (DEMs) and 268 edges (connectivity scores, CSs), ${ }^{30}$ which is a high-density small-world network (0.893) with a characteristic path length of 1.107 and a clustering coefficient of 0.927 (Fig. 5c and Supplementary Table S15), enriched for 88 GO-BPs and 16 KEGG pathways (Supplementary Fig. S5). Additionally, after determining the functions of the 25 targeted modules from the hub genes or the representative GO-BP and KEGG pathways, which were named "targeted functional modules" (TFMs), (Fig. 5c, Supplementary Tables S14, S16 and Fig. S5), we found that the 
$\square$ DHI group $(\mathrm{N}=585)$

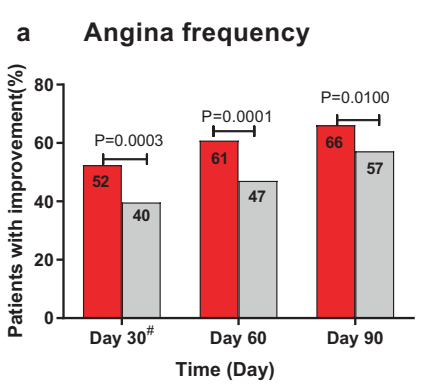

c Angina stability

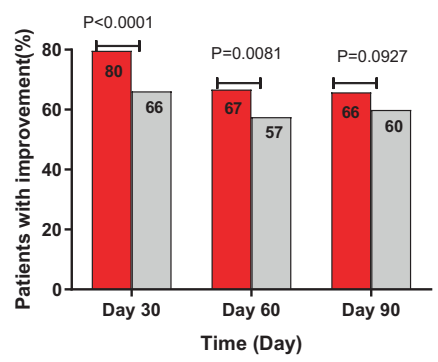

e Quality of life

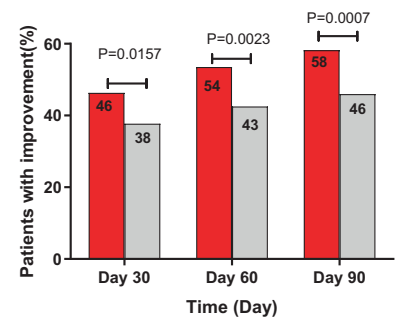

Control group $(\mathrm{N}=289)$

b Physical limitation

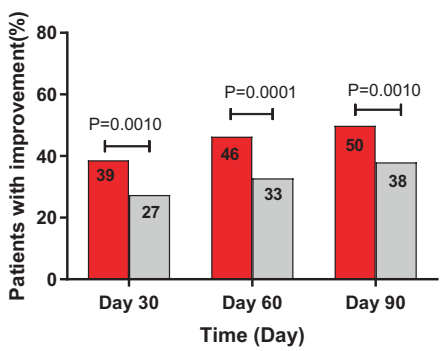

d Treatment satisfaction

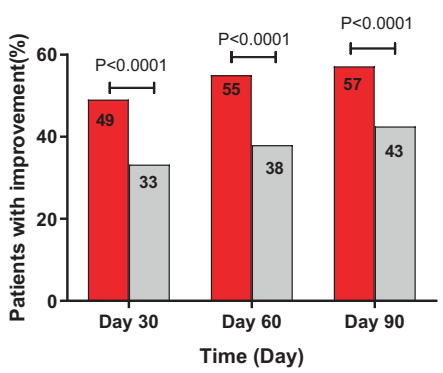

f

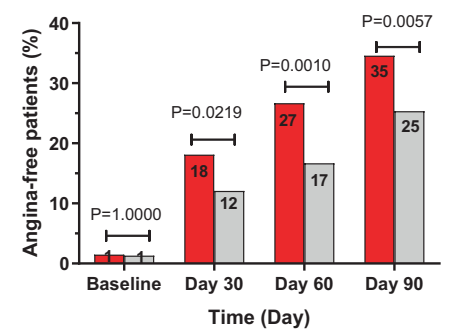
g $\underset{\substack{\mathrm{DH} \\ \text { Subgroup }}}{\substack{\mathrm{N}=613) \\ \text { Patients with improvement at Day } 30^{\#}}}$ Absolute Percentage-Point Different $(95 \% \mathrm{Cl}) \quad$ P-value

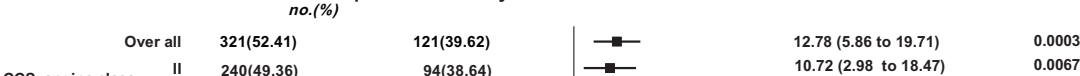
CCS angina class If $240(49.36) \quad 94(38.64)$

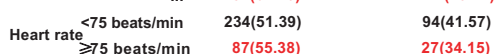

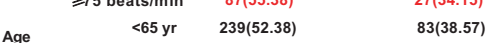
$\begin{array}{lll}\quad \geqslant 65 \mathrm{yr} & 82(52.50) & 38(42.19)\end{array}$ $\begin{array}{lrrr} & \text { Male } & 207(50.87) & 73(38.91) \\ & \text { Female } & 114(55.45) & 48(40.78)\end{array}$ History of Yes $\quad 73(57.98) \quad 17(29.19)$

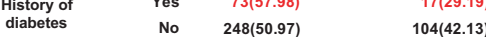
History of Yes 109(50.14)

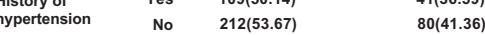
$\begin{array}{llrr}\text { History of } & \text { Yes } & \mathbf{2 8}(60.46) & \mathbf{8}(40.00)\end{array}$ hyperlipidemia $\quad$ No $293(51.70)$ Prior intensive Yes combination Yes 30415 medical therapy No 17(47.44) Nitroglycerin Yes 245(64.71) user No 76(32.50) Disease $\quad \leqslant 15 \mathrm{~d} \quad 308(53.32)$ duration of this $>15 \mathrm{~d} \quad 14(37.83)$ $113(39.60)$
$113(38.90)$ $113(38.90)$
$8(59.73)$

$$
\begin{aligned}
& 97(52.36) \\
& 23(19.72)
\end{aligned}
$$$$
\text { 114(41.05) }
$$$$
7(25.00)
$$

$0.72(2.98$ to 18.47$)$

0.0068

20.79 (5.73 to 35.85$)$

$9.82(1.77$ to 17.86$)$

13.81 (5.70 to 21.92$)$

$10.31(-2.92$ to 23.55$)$

$11.97(3.29$ to 20.64$) \quad 0.0068$

14.67 (3.26 to 26.09) $\quad 0.0118$

28.79 (13.93 to 43.65$) \quad 0.0001$

8.84 (1.08 to 16.60$)$

$13.55(2.13$ to 24.97$) \quad 0.0201$

12.30 (3.62 to 20.99) $\quad 0.0055$

20.46 (-5.48 to 46.39$) \quad 0.1221$

$12.16(4.98$ to 19.34$) \quad 0.0009$

$13.83(6.74$ to 20.91$) \quad 0.0001$

$\begin{array}{ll}-6.29(-37.17 \text { to } 24.60) & 0.6898\end{array}$

12.35 (3.51 to 21.19) $\quad 0.0062$

12.77 (3.17 to 22.38) $\quad 0.0092$

$\begin{aligned} 12.28(5.06 \text { to } 19.49) & 0.0009\end{aligned}$

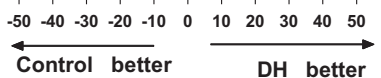

Fig. 2 Changes in Seattle Angina Questionnaire scores. a-e Patients with clinically significant improvements from baseline in their Seattle Angina Questionnaire scores; $\mathbf{f}$ Freedom from angina over time as assessed by the angina frequency scale of the Seattle Angina Questionnaire (scored out of 100); g Forest plot of the primary outcome in prespecified subgroups (at Day 30). "\#" in Figs. 1a and 1g indicates that the primary outcome was calculated from the imputed data, with $n=613$ in the DHI group and $n=305$ in the control group 
a Xueyu-Zheng score

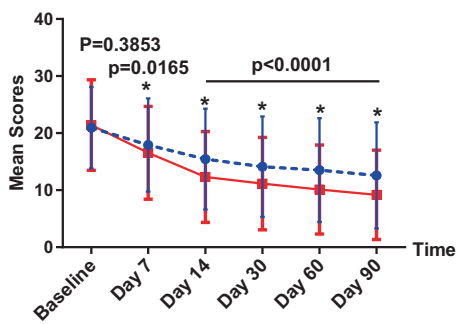

d Nitroglycerin consumption

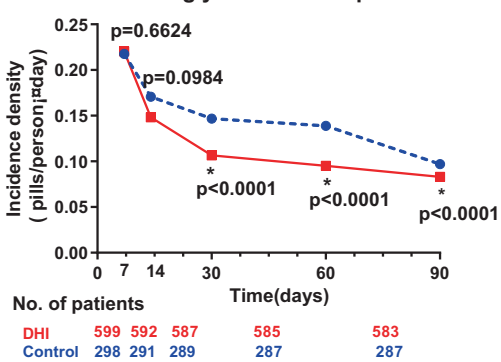

b Significant syndrome improvements in Xueyu-Zheng score

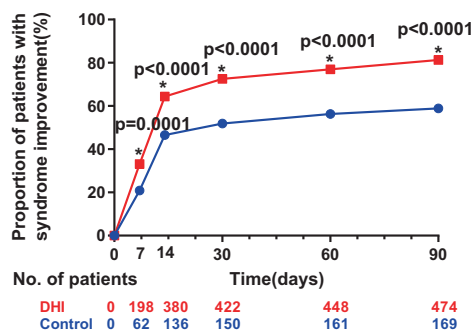

e Patients with improvement in CCS grading

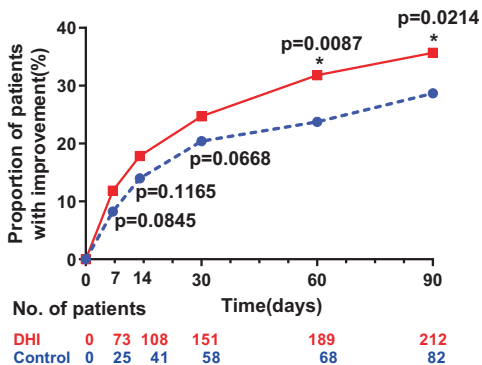

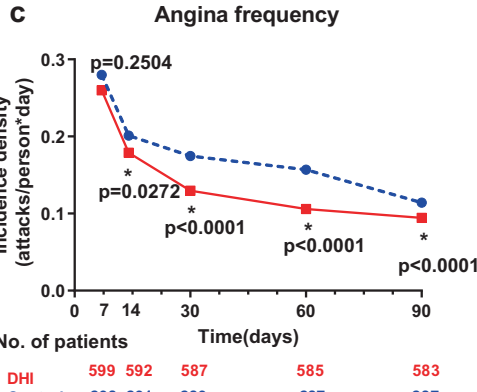

Control $\quad 298291 \quad 289 \quad 287 \quad 287$

f Triglyceride

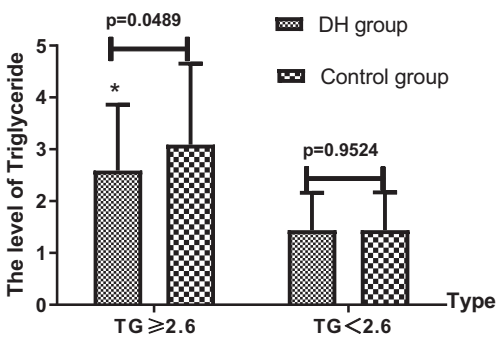

Fig. 3 Changes in other secondary outcomes between the two groups. a Comparison of mean Xueyu-Zheng scores at each time point. b The proportion of patients with significant improvements in Xueyu-Zheng scores (\%). c Changes in the incidence density of angina (attacks/personday) according to patients' diaries. $\mathbf{d}$ Changes in the incidence density of nitroglycerin consumption according to patients' diaries. e The proportion of patients with normal ECG recordings. $\mathbf{f}$ The proportion of patients with improved CCS grades

"signal transduction-PI3K-Akt signaling pathway" TFM was correlated with anti-inflammation, ${ }^{31}$ regulation of endothelial cell function ${ }^{32}$ and atherosclerotic plaque stability" ${ }^{31}$; the "miR-206 \& OR2F1" TFM is related to regulation of cholesterol metabolism ${ }^{33}$ and endothelial cell function. ${ }^{34}$

Globle flexibility of the effective therapeutic module in different responsed populations

Twelve, 37, and 46 DEMs were identified in the populations with the best effect $(\triangle A F \geq 40)$, a mild effect $(40>\Delta A F \geq 20)$ and no effect $(\triangle \mathrm{AF}<20)$, respectively (Fig. $6 \mathrm{a}, \mathrm{c}$ and Supplementary Fig. S6). The correlation of $90 \mathrm{DEGs}$ with $\triangle \mathrm{AF}$ at Day 30 was somewhat weak, ranging from -0.4574 to 0.3416 (Supplementary Table S17), which was significantly weaker than the correlation of DEMs with $\triangle \mathrm{AF}$ in the population with the best effect or a mild effect (Fig. 6b, d and Supplementary Fig. S7). The module with the highest correlation value $(0.909, p=0.0046)$ in the population with the best effect was identified as the most effective therapeutic module (METM) (Fig. 6d). This METM had 39 nodes, 741 edges and a high network density (Network density:1) (Fig. $6 e)$ and was related to the negative regulation of blood coagulation (GO:0030195, $p=0.0184$ ) (Supplementary Table S18). The topology of modules constructed with the genes in the METM was completely different among different populations (Fig. 6e). The D value, the global topological similarity to the METM, decreased as the effect increased $\left(r^{2}=0.8204, p=0.0019\right.$, Fig. $6 \mathrm{e}, \mathrm{f})$, while the number of edges in the flexible modules increased with the effect $\left(r^{2}=0.8115, p=0.0023\right.$, Fig. 6e, h). Six genes, ACSS3, SULT1A2, VTN, LRFN2, NPC1L1, and NEURL3, were identified as being highly significant to AF and having high module membership (Fig. 6f), which existed in the population with an effect $(\triangle A F \geq 10)$ but not in the population with no effect $(\triangle \mathrm{AF} \leq 0)$ (Fig. 6e). It has been reported that genetic variation in
NPC1L1 is associated with a reduction in the risk of ischemic vascular disease, with a corresponding reduction in LDL cholesterol. $^{35}$

\section{DISCUSSION}

In our study, we found that although $94.44 \%$ (867/918) patients had the prior optimal medical therapy for angina, they all had over moderate angina (CCS II-III), and even 189 patients encountered relatively severe angina (CCS III). Our study indicated that 14-day usage of $\mathrm{DHI}$ could significantly reduce the number of angina episodes and improve angina-specific health status for at least 90 days, particularly for patients with severe angina (CCS class III), faster heart rate (HR $\geq 75$ beats/min) or diabetes (Fig. $2 \mathrm{~g}$ ), who are most prone to refractory angina. We confirmed the additional benefit and safety of $\mathrm{DHI}$ when added to optimal medical therapy in patients with symchronic stable angina. The effect of the $\mathrm{DHI}$ in improving patients'stable CAD-specific health status was similar to that of percutaneous coronary intervention in the first 3 months. ${ }^{22}$ In addition, DHI did not bring any additional safety risk as an add-on therapy for patients with symptomatic angina.

This study is the first rigorously designed randomized controlled trial to evaluate the efficacy and safety of $\mathrm{DHI}$ in the management of symptomatic chronic stable angina. In this study, the central online randomization system, double blinding, the use of a placebo control and the role of the independent DMC may prevent potential selection, performance and detection biases. Therefore, the integrity and reliability of the data validate the concept that $\mathrm{DHI}$ is a promising therapeutic option for the treatment of chronic stable angina, which is also generally consistent with the findings of a previous small-sample clinical study. ${ }^{21}$

Furthermore, we firstly sketched the 25-targeted functional modular map and then detected a flexible effective therapeutic 

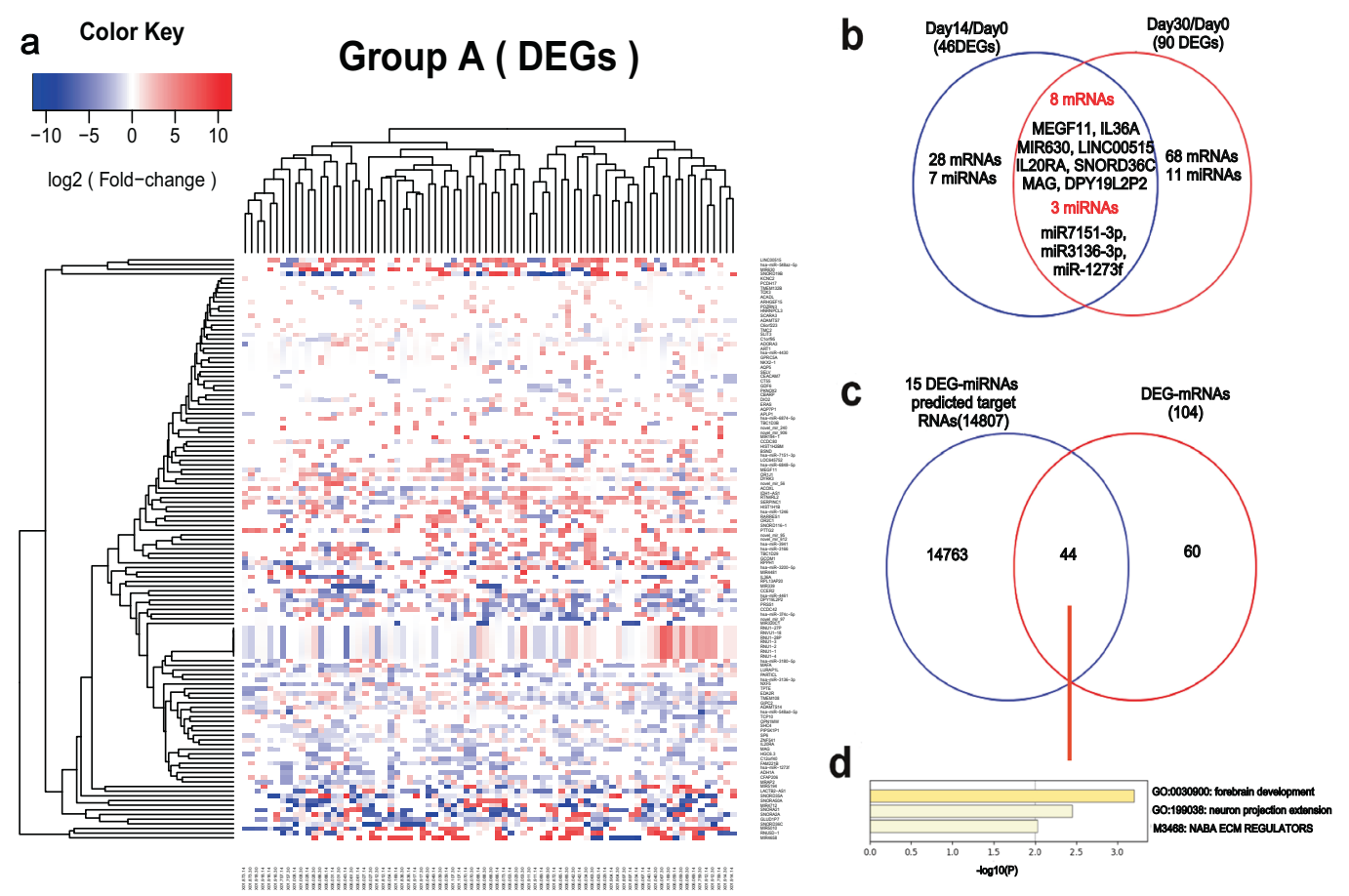

Fig. 4 Differentially express genes targeted by Danhong Injection. a Heatmap of the gene expression pattern (miRNA and mRNA) of the DHI group. Red boxes represent the genes that were upregulated after treatment, while blue ones represent downregulated. $\mathbf{b}$ Venn diagram of the numbers of differentially expressed genes (DEGs) before and after treatment. The overlapping area represents the number of genes found to be differentially expressed both at Day 14 or Day 30 after treatment. c Venn diagram of 104 DEG-mRNAs and the predicted target mRNAs of 15 DEG-mRNAs. d The GO biological process categories of the 44 overlapping DEG-mRNAs and the predicted target mRNAs of 15 DEG-mRNAs

module of $\mathrm{DHI}$ related to anticoagulation and the regulation of cholesterol metabolism ${ }^{35}$ with topological features that change with phenotype alteration, based on the analysis of DEMs rather than DEGs. We believe that the population with this module will achieve better drug effects than the population with any single DEG. The methodology of modular pharmacology used in our study was more suitable and efficient than network analysis of DEGs or pathways in uncovering the mechanism of multi-target drugs. As a module is generally considered a closely linked unit that performs biological functions at the network level, druginduced network rewiring may lead to changes in modular structure. ${ }^{36}$ Thus, analysis of DEMs must be especially useful in clarifying the mechanism of multi-target drugs and detecting the effective therapeutic module underlying their effects.

Besides, although millennia-old Chinese medicine treats disease with many combination therapies involving diverse ingredients used in clinic practice, the robust evidence for their effects and the underlying mechanism is far from enough. In our study, we provides an applicable paradigm for the future study of the similar Chinese medicine product with multi-targets on a certain disease with therapeutic advantages. It will be helpful to confirm the effect, uncover the therapeutic mechanism and facilitate the modernization of Chinese medicine.

We are also aware of a possible weakness of the study. Only 4 new-onset major vascular events and no deaths were reported in the $\mathrm{DHI}$ group during the 90-day follow-up; this period may not have been long enough to detect these endpoints and confirm the benefits of DHI. Future studies should be designed with longer follow-up periods.

In conclusion, an initial short-term course of $\mathrm{DH}$ along with optimal medical therapy relieved angina and improved selfassessed health status to a greater extent than an initial strategy of optimal medical therapy alone over a follow-up of approximately 90 days. The effect might correlate with an effective therapeutic module related to anticoagulation and the regulation of cholesterol metabolism. Our study provides a novel strategy to detect the effective therapeutic module of multi-target drugs based on modular flexibility in differentially responsive populations.

\section{MATERIALS AND METHODS}

\section{Study design}

This study was an adaptive-design, randomized, multicenter, double-blind, placebo-controlled trial. The trial protocol ${ }^{23}$ was approved by the central institutional review board from Chinese PLA General Hospital (IRB No. [2012] Pharmaceutical (025)). This trial complied with the principles of the Declaration of Helsinki. Informed written consent was obtained from all participants. Patients or the public were not involved in the design, or conduct, or reporting, or dissemination plans of our research.

\section{Participants}

Symptomatic patients aged $18-70$ years who were diagnosed with CAD and "Xueyu-Zheng", a score of at least 15 on the Chinese Medicine Symptom Scale of "Xueyu-Zheng" for angina patients, ${ }^{23}$ and had CCS class II or III angina were enrolled in this study. Key exclusion criteria included a history of myocardial infarction within the past 3 months; severe complications such as liver or renal dysfunction, severe cardiopulmonary dysfunction, etc.; a history of epilepsy or cerebral hemorrhage; a history of drug-induced bleeding or hematopoietic disorder; patients who underwent a surgery within the past 4 weeks or those who had a hemorrhagic tendency.

\section{Interventions}

Optimal medical therapy was given to all participants throughout the trial, including antiplatelet agents (aspirin and/or clopidogrel), lipid-lowering agents (atorvastatin or simvastatin) and anti-angina 
a Cluster dendrogram (Danhong group at Day 30)
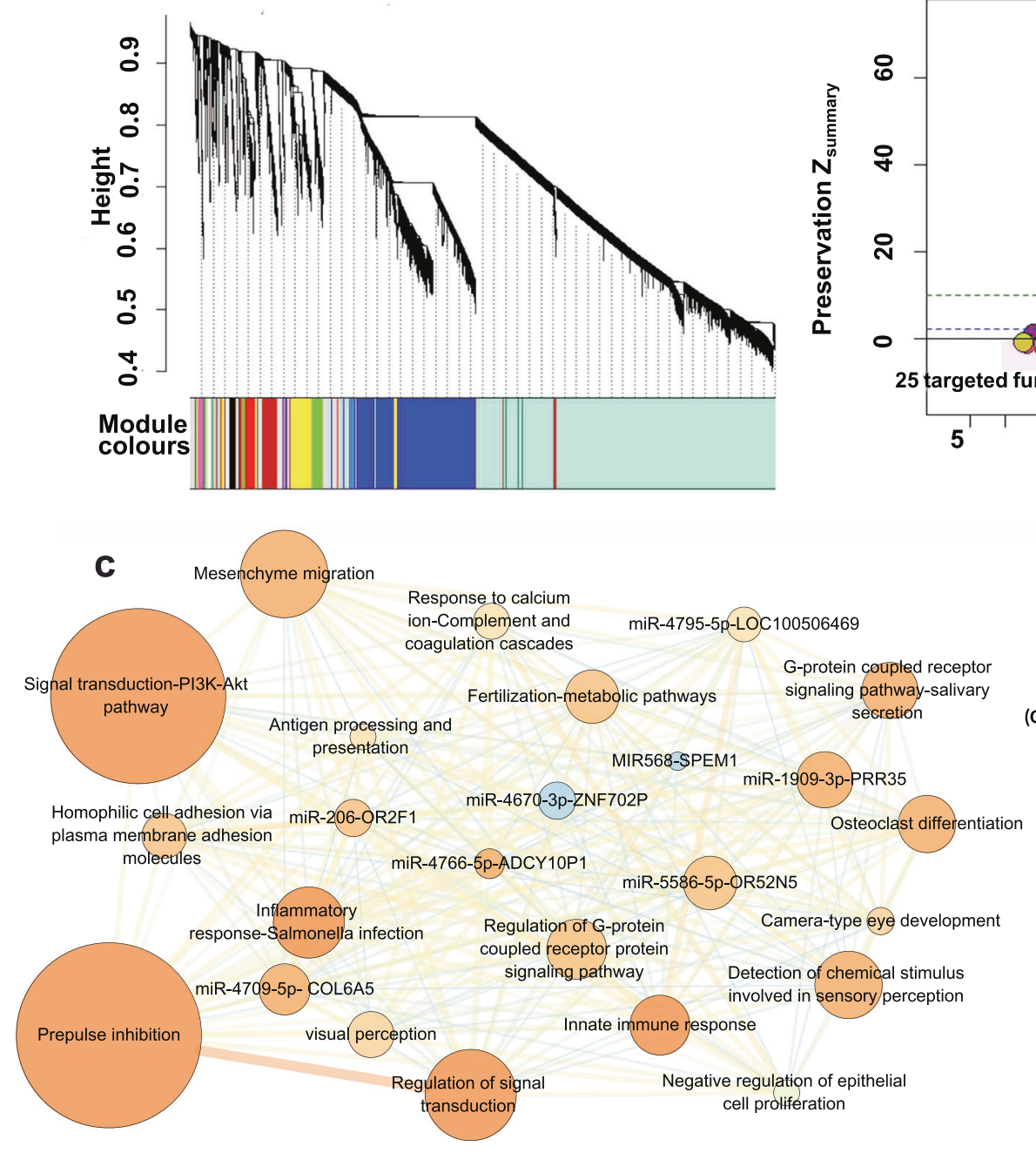

b
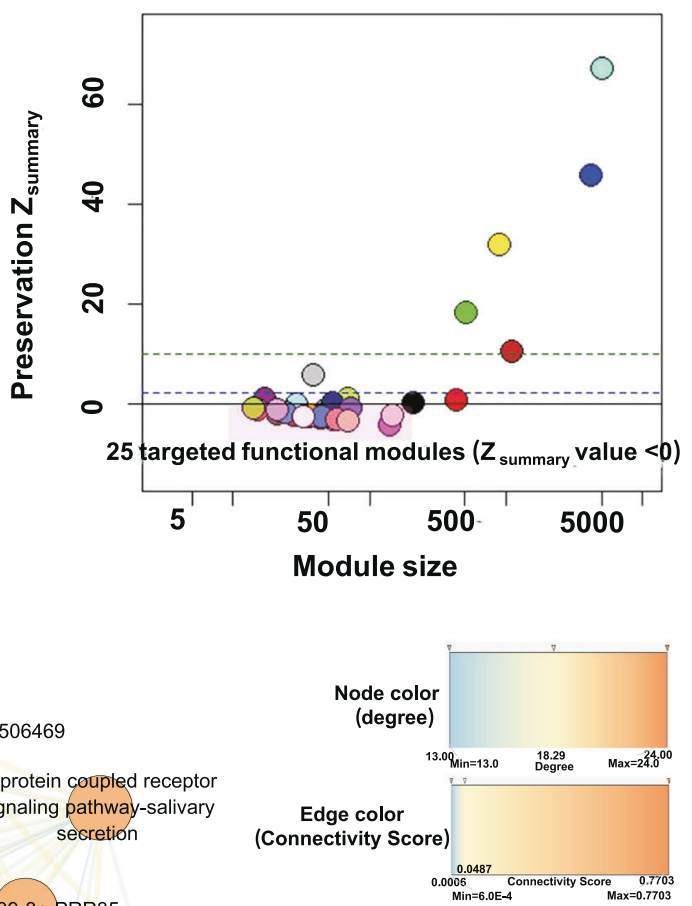

Network topology parameters: Clustering Coefficient: $\mathbf{0 . 9 2 7}$ Connected Components: 1

Network diameter: 2 Network radius: 1

Network Centralization: $\mathbf{0 . 1 1 6}$

Shortest paths: $600(100 \%)$

Characteristic path length: 1.107

Avg. number of neighbors: 21.44

Number of nodes: 25

Network density: $\mathbf{0 . 8 9 3}$

Network heterogeneity: 0.138

Isolated nodes: 0

Number of self-loops: 0

Multi-edge node pairs: 0

Fig. 5 Targeted modular map by the multi-target drug Danhong Injection. a The hierarchic cluster tree (dendrogram) of DHI. Each major tree branch represents a module, and each module is labeled with a color below the dendrogram. $\mathbf{b}$ The distribution of modules by $Z_{\text {summary }}$ value. There were 25 differentially expressed modules $(D E M s)$ with negative $Z_{\text {summary }}$ values $(<0)$; these modules were considered the targeted functional modules (TFMs). c The 25-TFM map for DHI at Day 30 with the 25 TFMs as nodes and the connectivity score (CS) as the edge between each pair of modules. The node size indicates the number of genes in the corresponding TFM, and the color of the node shows the degree of the TFM in the network

agents ( $\beta$-blockers, long-acting nitrates, or calcium channel blockers). All of these basic treatments were recorded in detail on electronic case report forms (eCRFs). Participants in the DHI and control groups received a daily intravenous drip of $40 \mathrm{ml} \mathrm{DHI}$ and $40 \mathrm{ml}$ placebo ( $0.9 \%$ normal saline), respectively, added to $250 \mathrm{ml}$ $0.9 \%$ normal saline. All participants underwent 2 weeks of treatment and 76 days of follow-up. Patients could take a nitroglycerin tablet ( $0.5 \mathrm{mg}$ per tablet, provided by Beijing Yimin Pharmaceutical Co., Ltd.) in the event of an angina attack, and they recorded the details regarding the angina attack and the usage of nitroglycerin in a patient diary. Other drugs with the same indications as $\mathrm{DHI}$ were not allowed to be used throughout the study period. All concomitant medications were recorded on the eCRFs.

\section{Outcome measurements}

Angina-specific health status was assessed at baseline and on days 30,60, and 90. Each assessment was performed using the $\mathrm{SAQ}$, a 19-item questionnaire with 5 domains, namely, physical limitation ( $P L)$, angina stability (AS), angina frequency (AF), treatment satisfaction (TS), and quality of life (QL), each scored on a scale from 0 to 100 , where a higher score indicates a better health status (13). A clinically significant change on the SAQ was defined as a difference of 8 points or more on $\mathrm{PL}, 25$ or more on $\mathrm{AS}, 20$ or more on $\mathrm{AF}, 12$ or more on TS, or 16 or more on QL. ${ }^{22}$ The primary outcome was the proportion of patients with a clinically significant change in SAQ-AF at Day 30 . The proportions of patients who experienced clinically significant changes in the other four SAQ domains were used as secondary outcome measures. Other secondary outcomes included the Chinese Medicine Symptom Scale of "Xueyu-Zheng"; ${ }^{23}$ frequency of angina attacks; consumption of short-acting nitrates; improvements in CCS class and electrocardiogram recordings; changes in biochemical indexes such as serum lipids, hs-CRP or CRP and PAR at Day 14; the incidence of new-onset major vascular events; overall mortality within 90 days; the frequency of adverse events (AEs); and the incidence of moderate or severe hemorrhages within 90 days, details are provided in the protocol. $^{23}$

Another outcome measure, the change of total exercise duration (TED) in exercise tolerance test (ETT) from baseline to 

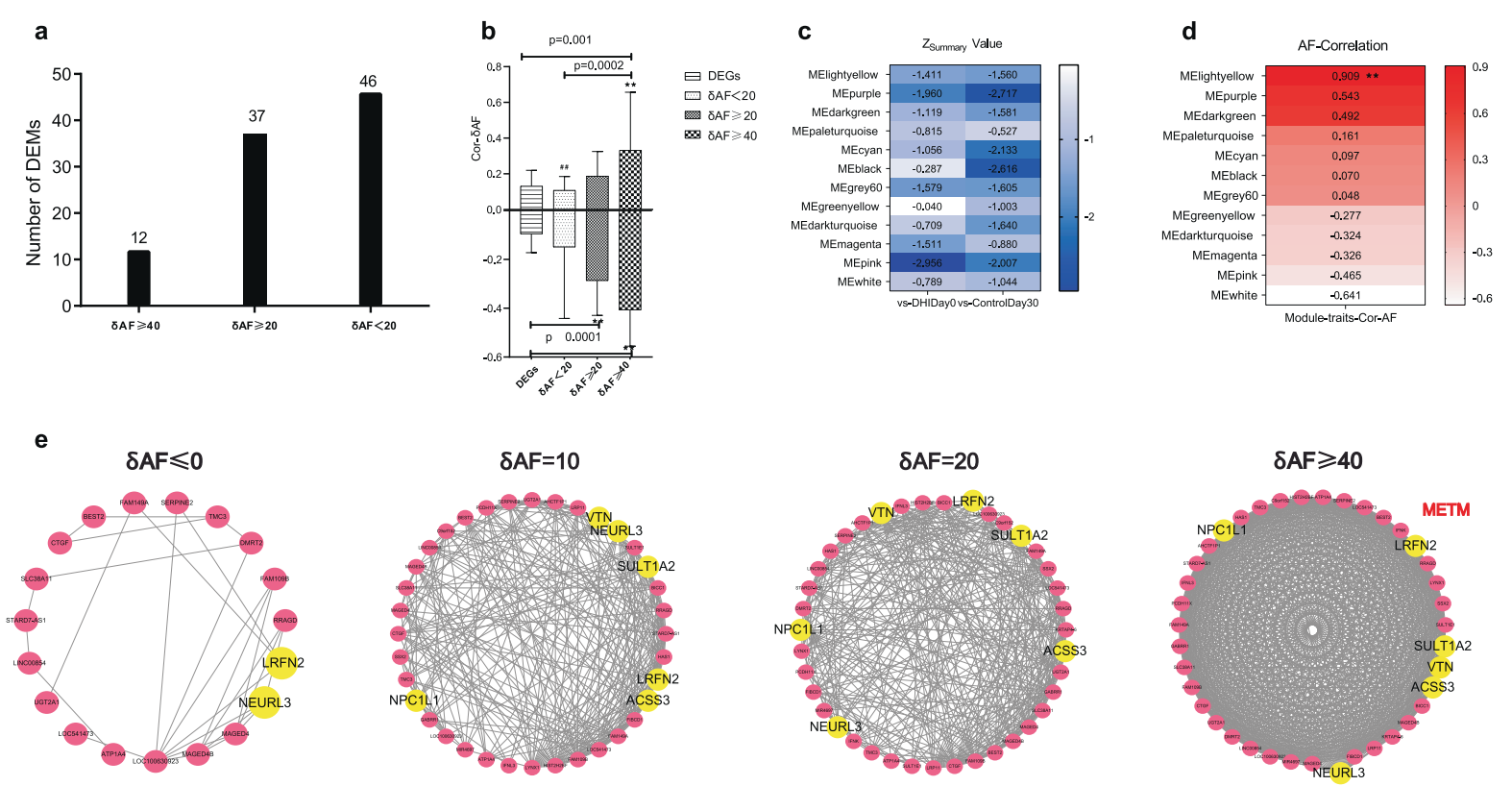

\begin{tabular}{|c|c|c|c|c|c|c|c|c|c|c|c|}
\hline $\begin{array}{l}\text { Popul } \\
\text { ations }\end{array}$ & $\begin{array}{l}\text { Nod } \\
\text { es }\end{array}$ & $\begin{array}{c}\text { Edg } \\
\text { es }\end{array}$ & $\begin{array}{l}\text { Average } \\
\text { degree }\end{array}$ & $\begin{array}{l}\text { Network } \\
\text { density }\end{array}$ & $\begin{array}{l}\text { Network } \\
\text { hetero- } \\
\text { geneity }\end{array}$ & $\begin{array}{l}\text { Clustering } \\
\text { coefficient }\end{array}$ & $\begin{array}{c}\text { Average } \\
\text { shortest } \\
\text { path length }\end{array}$ & $\begin{array}{c}\text { Network } \\
\text { centraliza- } \\
\text { tion }\end{array}$ & $\begin{array}{c}\text { Between- } \\
\text { ness } \\
\text { centrality }\end{array}$ & $\begin{array}{l}\text { Closeness } \\
\text { centrality }\end{array}$ & $\begin{array}{c}\text { D- } \\
\text { value }\end{array}$ \\
\hline$\delta A F \leq 0$ & 19 & 27 & 2.842 & 0.158 & 0.585 & 0.427 & 3.275 & 0.32 & 0.134 & 0.323 & 3.162 \\
\hline $\begin{array}{c}\delta A F= \\
10\end{array}$ & 36 & 272 & 15.111 & 0.432 & 0.309 & 0.583 & 1.602 & 0.208 & 0.018 & 0.631 & 1.717 \\
\hline $\begin{array}{c}\delta A F= \\
20\end{array}$ & 39 & 337 & 17.282 & 0.455 & 0.222 & 0.598 & 1.547 & 0.159 & 0.015 & 0.649 & 1.519 \\
\hline $\begin{array}{c}\delta A F \geq \\
40\end{array}$ & 39 & 741 & 38 & 1 & 0 & 1 & 1 & 0 & 0 & 1 & 0 \\
\hline
\end{tabular}

f

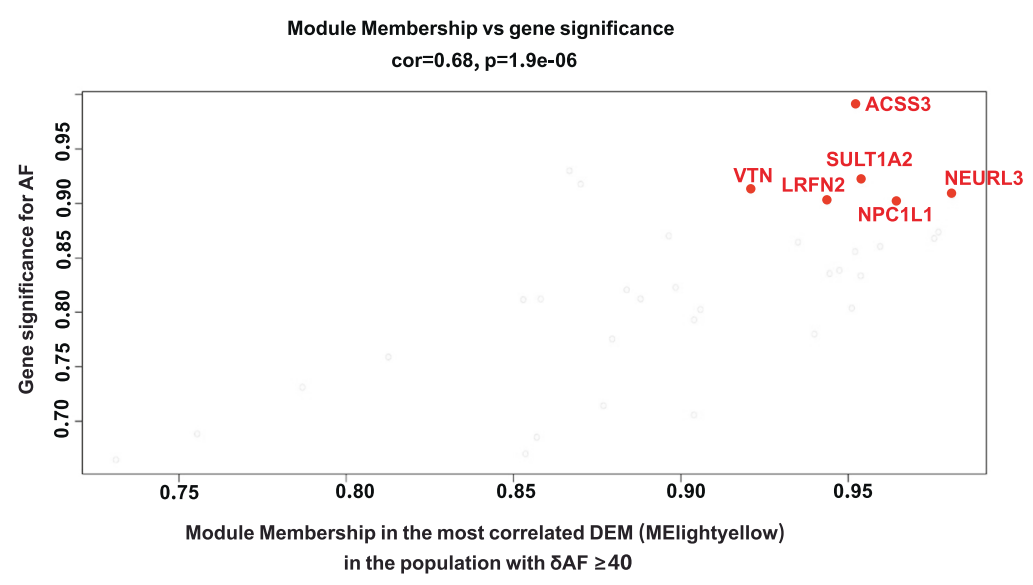

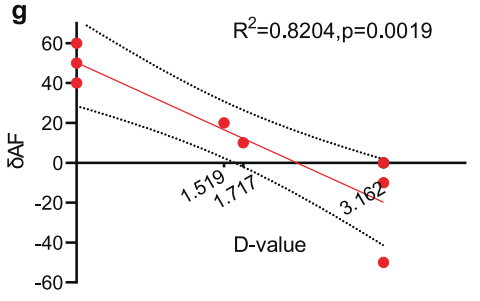

h

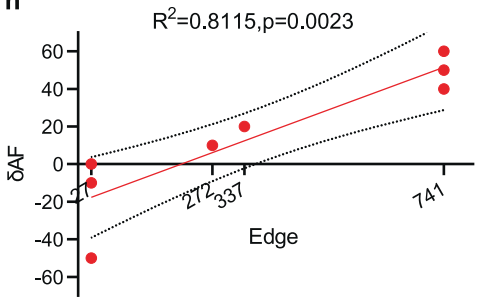

Day 14, was assessed in 290 patients until the first interim analysis. However, after the first interim analysis, the Data Monitoring Committee (DMC) recommended that the ETT test be omitted for better patient compliance and higher feasibility of the trial.
Randomisation and blinding

Eligible participants were randomly assigned to either the $\mathrm{DHI}$ group or the control group in a 2:1 ratio, with a block size of 6, via a central randomization system (Brightech, Somerset, USA) This system automatically randomized patients and generated a 
Fig. 6 The effective target module detected in the responsive population. a The numbers of differentially expressed modules (DEMs) with negative Zsummary values $(<0)$ according to WGCNA in the populations with different responses to $\mathrm{DHI}$ treatment at Day 30 . $\triangle \mathrm{AF}$ indicates the change in the Seattle Angina Questionnaire angina frequency scale (SAQ-AF) score from baseline (Day 0) to Day 30. b Comparison of the correlation with $\triangle \mathrm{AF}$ among DEGs and DEMs in different populations. The significance of the difference between each pair of groups was calculated by ANOVA; ** indicates the notable statistical significance of the correlation with $\triangle \mathrm{AF}$ between DEGs and the DEMs in the populations with $\triangle A F \geq 40$, and \#\# indicates the notable statistical significance of the correlation with $\triangle A F$ between the DEMs in the populations with $\Delta \mathrm{AF} \leq 0$ and $\Delta \mathrm{AF} \geq 40$. c Heatmap of the Zsummary value pattern of the $12 \mathrm{DEMs}$ at Day 30 in the populations with $\Delta \mathrm{AF} \geq 40$ compared with the baseline (Day 0 ) and the control group. $\mathbf{d}$ Heatmap of correlation with $\triangle \mathrm{AF}$ for the 12 DEMs at Day 30 in the populations with $\triangle \mathrm{AF} \geq 40$. e The modular flexibility of the effective therapeutic module in the populations with different responses to $\mathrm{DHI}$ treatment. The topological parameters are shown in the table. $f$ The gene distribution in the effective therapeutic module shows the correlation between gene significance to $\mathrm{AF}$ and module membership. $\mathbf{g}, \mathbf{h}$ The linear regression correlation between $\mathrm{D}$ value $(\mathbf{g})$ or edge (h) and $\Delta \mathrm{AF}$

randomization number with a message noting their assigned treatment. Randomization was stratified based on whether a patient received standard conventional therapy for more than 1 week prior to study initiation.

Since the color of $\mathrm{DHI}$ and $0.9 \%$ saline are different, the dropping bottles were wrapped in sealed shaded bags, and brown infusion devices were used for infusion. These procedures were implemented by two professional nurses who were required to sign a confidentiality agreement before study initiation and not to contact each other. One of the professional nurses was in charge of preparing the drugs in a special transfusion room and sealing the infusion bottles with shaded brown bags. The other nurse took the prepared drugs from the transfusion room to the infusion nurse and supervise the infusion process to ensure that the allocation of the drugs is blinded to the patients. The participants, outcome assessors, and statisticians were blinded to treatment allocation.

\section{Statistical analysis}

Using EAST5.2, we initially estimated that at least 870 patients needed to be enrolled in the study in order to achieve $85 \%$ power to detect a $10 \%$ difference in the primary outcome between the $\mathrm{DHI}$ and control groups at Day $30,{ }^{22}$ given a significance level of $5 \%$ and a dropout rate of $20 \%$. Two interim analyses with O'Brien-Fleming ${ }^{37}$ stopping boundaries were performed to re-estimate the sample size after 288 and 582 patients completed the trial. The sample size was adjusted to 920 by DMC after the second interim analysis.

The primary outcome was analyzed according to the intention-totreat principle. Missing data were handled by regression-based multiple imputation using the fully conditional method. ${ }^{38}$ Three sensitivity analyses ( 1 preplanned and two post hoc) were conducted for the primary outcome. The preplanned used controlbased pattern model to evaluate sensitivity to missing data departure from the missing at random assumption. Two post hoc sensitivity analyses evaluated whether baseline imbalance in clinical site and a mixed-effect model with repeated measures could have diluted the estimates of treatment effect. Continuous variables were reported as the mean and standard deviation (SD), and categorical variables were reported as frequencies and percentages with $95 \%$ Cls. Statistical differences between groups were analyzed using Student's $t$-test or the Wilcoxon rank-sum test for quantitative data and chi-squared test for categorical data. All reported $P$ values were two-sided, and a $P$ value less than 0.05 was considered to be statistically significant except in the primary outcome analyses, in which Bonferroni's multiple comparison test was applied twice and $P<0.016$ was considered significant. All calculations were performed using SAS 9.4 (SAS Institute Inc., USA).

\section{Analysis of RNA profile and targeted modules}

The serum samples of patients from 301 Hospital and Xuanwu Hospital were sequenced using the Illumina HiSeq sequencing platform according to the manufacturer's instructions and previous studies described. ${ }^{39,40}$ Significant differentially expressed genes (DEGs) were defined as genes with a $P$ value $<0.05$ and at least a 1.5 -fold change. The construction of the gene coexpression network for $\mathrm{DHI}$ at Day 30 and the identification of modules were implemented using WGCNA, an R package for weighted correlation network analysis. ${ }^{28} \mathrm{~A}$ module with a $Z_{\text {summary }}$ value less than 0 compared with the control group was defined as a differentially expressed module (DEM). ${ }^{29}$ Furthermore, we reconstructed the targeted DEM network using the connectivity score $(C S)^{30}$ among these modules. Each targeted DEM was assigned a certain function, using its hub genes with the highest degree or the most significant Gene Ontology (GO) and Kyoto Encyclopedia of Genes and Genomes (KEGG) pathways enriched using the Database for Annotation, Visualization and Integrated Discovery (DAVID) Bioinformatics Resources $6.8 i^{41}$ these modules were designated as targeted functional modules (TFMs). To further determine the molecular features of the targeted population, we divided the populations in the $\mathrm{DH}$ group at Day 30 into 3 groups according to the phenotype alteration, $\triangle \mathrm{AF}$, defined as the change in SAQ-AF at Day 30 from baseline: the group with the best effect $(\triangle A F \geq 40)$, a group with a mild effect $(40>\Delta \mathrm{AF} \geq 20)$ and a group with no effect $(\Delta \mathrm{AF}<20)$. Significant DEMs were defined as those with a correlation value $(r) \geq$ 0.3 and a $P$ value $<0.05$ as calculated by WGCNA, 28 and the most significant DEM (with the highest $r$ and lowest $P$ value) in the population with the best effect was considered the most effective therapeutic module (METM) of DHI. The flexibility of the METM in differentially responsive populations was assessed with the global topological parameter $\mathrm{D}$, based on Euclidean distance. ${ }^{42,43}$

\section{DATA AVAILABILITY}

The data that support the findings of this study have been deposited in the CNSA (https://db.cngb.org/cnsa/) of CNGBdb with accession number CNP0000461.

\section{ACKNOWLEDGEMENTS}

We thank all the staff of the 31 clinical centers for their contributions to the investigation of the trial. This work was funded by China National Science and Technology Major Project for "Significant New Drugs Development" (2011ZX09304-07), National Natural Science Foundation of China (81673833), and China Fundamental Research Funds for the Central Public Welfare Research Institutes (ZZ0908029).

\section{AUTHOR CONTRIBUTIONS}

Conceptualization: Yun-Dai Chen, Yong-Yan Wang, Zhong Wang. Data curation: Bing Wei Chen. Formal analysis: Bing-Wei Chen, Jun Liu' ${ }^{1}$, Fei Teng, Wang-Min Qiao, XiaoLong Zhu, Zhong Wang. Methodology: Chun-Ti Shen, Qi-Guang Chen, Bing-Wei Chen, Zhong Wang, Yong-Yong Wang. Writing-original draft: Jun Liu'. Writing-critical review \& editing: all authors. Investigation: Yun-Dai Chen, Dan-Dan Li, Wei Dong, YuQi Liu, Yang Wu, Da-Xuan Tang, Fu-Chun Zhang, Meng Qiu, Qi Hua, Jing-Yu He, Jun Li, Bai Du, Ting-Hai Du, Lin-Lin Niu, Xue-Jun Jiang, Bo Cui, Jiang-Bin Chen, Yang-Gan Wang, Hai-Rong Wang, Qin Yu, Jing He, Yi-Lin Mao, Xiao-Fang Bin, Yue Deng, Yu-Dan Tian, Qing-Hua Han, Da-Jin Liu, Li-Qin Duan, Ming-Jun Zhao, Cui-Ying Zhang, Hai-Ying Dai, Ze-Hua Li, Ying Xiao, You-Zhi Hu, Xiao-Yu Huang, Kun Xing, Xin Jiang, Chao-Feng Liu, Jing An, Feng-Chun Li, Tao Tao, Jin-Fa Jiang, Ying Yang, Yao-Rong Dong, Lei Zhang, Guang Fu, Ying Li ${ }^{22}$, Shu-Wei Huang, Li-Ping Dou, Lan-Jun Sun, Ying-Qiang Zhao, Jie Li, Yun Xia, Jun Liu ${ }^{25}$, Fan Liu, Wen-Jin He, Ying $\mathrm{Li}^{26}$, Jian-Cong Tan, Yang Lin, Ya-Bin Zhou, Jian-Fei Yang, Guo-Qing Ma, Hui-Jun Chen, He-Ping Liu, Zong-Wu Liu, Jian-Xiong Liu, Xiao-Jia Luo, Xiao-Hong Bin. Funding acquisition: Zhong Wang, YongYan Wang, Jun Liu'. Project administration: Yong-Yan Wang, Zhong Wang, Yun-Dai Chen. Supervision: Chun-Ti Shen, Qi-Guang Chen, Yong-Yan Wang. 


\section{ADDITIONAL INFORMATION}

Supplementary information The online version contains supplementary material available at https://doi.org/10.1038/s41392-021-00741-x.

Competing interests: The authors declare no competing interests.

\section{REFERENCES}

1. Mozaffarian, D. et al. Heart disease and stroke statistics-2015 update: a report from the American Heart Association. Circulation 131, e29-e322 (2015).

2. Jain, K. K. Personalized management of cardiovascular disorders. Med. Princ. Pract. 26, 399-414 (2017).

3. Mathur, S. \& Sutton, J. Personalized medicine could transform healthcare. Biomed. Rep. 7, 3-5 (2017).

4. Johnson, K. W. et al. Enabling precision cardiology through multiscale biology and systems medicine. Jacc. Basic. Transl. Sci. 2, 311-327 (2017).

5. Chrysant, S. G. \& Chrysant, G. S. Future of polypill use for the prevention of cardiovascular disease and strokes. Am. J. Cardiol. 114, 641-645 (2014).

6. Zheng, P. F. et al. Genes associated with inflammation may serve as biomarkers for the diagnosis of coronary artery disease and ischaemic stroke. Lipids Health Dis. 19, 37 (2020).

7. He, J., Li, X., Zhang, Y., Zhang, Q. \& Li, L. Comprehensive analysis of ceRNA regulation network involved in the development of coronary artery disease. Biomed. Res. Int. 2021, 6658115 (2021).

8. Wang, L., Wang, Y., Hu, Q. \& Li, S. Systematic analysis of new drug indications by druggene-disease coherent subnetworks. CPT Pharmacomet. Syst. Pharm. 3, e146 (2014).

9. Ge, J. et al. Personalized early-warning signals during progression of human coronary atherosclerosis by landscape dynamic network biomarker. Genes (Basel). 11, 676 (2020).

10. Cohain, A. T. et al. An integrative multiomic network model links lipid metabolism to glucose regulation in coronary artery disease. Nat. Commun. 12, 547 (2021).

11. Wang, Z., Liu, J., Yu, Y., Chen, Y. \& Wang, Y. Modular pharmacology: the next paradigm in drug discovery. Expert Opin. Drug. Discov. 7, 667-677 (2012).

12. Wang, Z. \& Wang, Y. Navigating personalized medicine dependent on modular flexibility. Trends Mol. Med. 19, 393-395 (2013).

13. Li, X. L. et al. Postmarketing safety surveillance and reevaluation of Danhong injection: clinical study of 30888 cases. Evid. Based. Complement. Alternat. Med. 2015, 610846 (2015).

14. Feng, X. et al. Danhong injection in cardiovascular and cerebrovascular diseases: pharmacological actions, molecular mechanisms, and therapeutic potential. Pharmacol. Res. 139, 62-75 (2019).

15. Hu, Z. et al. Danhong injection mobilizes endothelial progenitor cells to repair vascular endothelium injury via upregulating the expression of Akt, eNOS and MMP-9. Phytomedicine 61, 152850 (2019).

16. Zhou, M. et al. Danhong injection attenuates high-fat-induced atherosclerosis and macrophage lipid accumulation by regulating the PI3K/AKT insulin pathway. J. Cardiovasc Pharm. 74, 152-161 (2019).

17. Fan, H. et al. Effects of Danhong injection on platelet aggregation in hyperlipidemia rats. J. Ethnopharmacol. 212, 67-73 (2018).

18. Zhang, L. et al. Dan Hong injection protects against cardiomyocytes apoptosis by maintaining mitochondrial integrity through Keap $1 /$ nuclear factor erythroid 2-related factor 2/JNK pathway. Front Pharm. 11, 591197 (2020).

19. Li, Z. M., Xu, S. W. \& Liu, P. Q. Salvia miltiorrhizaBurge (Danshen): a golden herbal medicine in cardiovascular therapeutics. Acta Pharmacologica Sin. 39, 802-824 (2018).

20. $\mathrm{Yu}, \mathrm{C}$. et al. Effects of danshensu on platelet aggregation and thrombosis: in vivo arteriovenous shunt and venous thrombosis models in rats. PLOS ONE 9, e110124 (2014).

21. Sun, K., Fu, C., Nie, S. \& You, Y. The index and improvement effect of using Danhong injection to patients with atherosclerosis symptoms of coronary heart disease (CHD). Pak. J. Pharm. Sci. 27, 1699-1704 (2014).

22. Weintraub, W. S. et al. Effect of $\mathrm{PCl}$ on quality of life in patients with stable coronary disease. N. Engl. J. Med. 359, 677-687 (2008).
23. Wang, P. Q. et al. Danhong injection in the treatment of chronic stable angina: study protocol for a randomized controlled trial. Trials 16, 474 (2015).

24. Griffiths-Jones, S., Saini, H. K., van Dongen, S. \& Enright, A. J. miRBase: tools for microRNA genomics. Nucleic Acids Res. 36, D154-D158 (2008).

25. Friedländer, M. R., Mackowiak, S. D., Li, N., Chen, W. \& Rajewsky, N. miRDeep2 accurately identifies known and hundreds of novel microRNA genes in seven animal clades. Nucleic, Acids Res. 40, 37-52 (2012).

26. Agarwal, V., Bell, G. W., Nam, J. W. \& Bartel, D. P. Predicting effective microRNA target sites in mammalian mRNAs. Elife 4, e05005 (2015).

27. Zhou, Y. et al. Metascape provides a biologist-oriented resource for the analysis of systems-level datasets. Nat. Commun. 10, 1523 (2019).

28. Langfelder, P. \& Horvath, S. WGCNA: an R package for weighted correlation network analysis. BMC Bioinforma. 9, 559 (2008).

29. Li, B. et al. Quantitative identification of compound-dependent on-modules and differential allosteric modules from homologous ischemic networks. Cpt. Pharmacomet. Syst. Pharmacol. 5, 575-584 (2016).

30. Levy, O., Knisbacher, B. A., Levanon, E. Y. \& Havlin, S. Integrating networks and comparative genomics reveals retroelement proliferation dynamics in hominid genomes. Sci. Adv. 3, e1701256 (2017).

31. Yan, H. et al. Insulin inhibits inflammation and promotes atherosclerotic plaque stability via PI3K-Akt pathway activation. Immunol. Lett. 170, 7-14 (2016).

32. Wang, N. et al. Overexpression of CREG attenuates atherosclerotic endothelium apoptosis via VEGF/PI3K/AKT pathway. Atherosclerosis 218, 543-551 (2011).

33. Vinod, M. et al. miR-206 controls LXRa expression and promotes LXR-mediated cholesterol efflux in macrophages. Biochim. Biophys. Acta 1841, 827-835 (2014).

34. Tang, Y., Zhang, Y., Chen, Y., Xiang, Y. \& Xie, Y. Role of the microRNA, miR-206, and its target PIK3C2a in endothelial progenitor cell function-potential link with coronary artery disease. FEBS J. 282, 3758-3772 (2015).

35. Lauridsen, B. K., Stender, S., Frikke-Schmidt, R., Nordestgaard, B. G. \& TybjærgHansen, A. Genetic variation in the cholesterol transporter NPC1L1, ischaemic vascular disease, and gallstone disease. Eur. Heart J. 36, 1601-1608 (2015).

36. Luscombe, N. M. et al. Genomic analysis of regulatory network dynamics reveals large topological changes. Nature 431, 308-312 (2004).

37. O'Brien, P. C. \& Fleming, T. R. A multiple testing procedure forclinical trials. Biometrics 35, 549-556 (1979).

38. Little, R. J. et al. The prevention and treatment of missing data in clinical trials. $N$. Engl. J. Med. 367, 1355-1360 (2012).

39. Meder, B. et al. Influence of the confounding factors age and sex on microRNA profiles from peripheral blood. Clin. Chem. 60, 1200-1208 (2014).

40. Leidinger, P. et al. A blood based 12-miRNA signature of Alzheimer disease patients. Genome Biol. 14, R78 (2013).

41. Huang, D. W., Sherman, B. T. \& Lempicki, R. A. Systematic and integrative analysis of large gene lists using DAVID Bioinformatics Resources. Nat. Protoc. 4, 44-57 (2009).

42. Wang, B. et al. Similarity network fusion for aggregating data types on a genomic scale. Nat. Methods 11, 333-337 (2014).

43. Nguyen, V. A. \& Lió, P. Measuring similarity between gene expression profiles: a Bayesian approach. Bmc. Genomics. 10, S14 (2009).

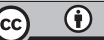

Open Access This article is licensed under a Creative Commons Attribution 4.0 International License, which permits use, sharing, adaptation, distribution and reproduction in any medium or format, as long as you give appropriate credit to the original author(s) and the source, provide a link to the Creative Commons license, and indicate if changes were made. The images or other third party material in this article are included in the article's Creative Commons license, unless indicated otherwise in a credit line to the material. If material is not included in the article's Creative Commons license and your intended use is not permitted by statutory regulation or exceeds the permitted use, you will need to obtain permission directly from the copyright holder. To view a copy of this license, visit http://creativecommons. org/licenses/by/4.0/.

(c) The Author(s) 2021 